\title{
Sleep is associated with task-negative brain activity in fibromyalgia participants with comorbid chronic insomnia
}

This article was published in the following Dove Press journal:

Journal of Pain Research

12 November 2015

Number of times this article has been viewed

\author{
Karlyn E Vatthauer' \\ Jason G Craggs' \\ Michael E Robinson' \\ Roland Staud ${ }^{2}$ \\ Richard B Berry² \\ William M Perlstein' \\ Christina S McCrae' \\ 'Department of Clinical and Health \\ Psychology, University of Florida, \\ Gainesville, FL, USA; ${ }^{2}$ Department \\ of Medicine, University of Florida, \\ Gainesville, FL, USA
}

Correspondence: Christina S McCrae Department of Health Psychology, School of Health Professions, University of Missouri, One Hospital Drive, Dcl 16 88, Columbia, MO 652/2, USA

Tel + I 573882 I56 I

Fax +I 5738841889

Email mccraec@health.missouri.edu

\begin{abstract}
Patients with chronic pain exhibit altered default mode network (DMN) activity. This preliminary project questioned whether comorbid disease states are associated with further brain alterations. Thirteen women with fibromyalgia (FM) only and 26 women with fibromyalgia with comorbid chronic insomnia (FMI) underwent a single night of ambulatory polysomnography and completed a sleep diary each morning for 14 days prior to performing a neuroimaging protocol. Novel imaging analyses were utilized to identify regions associated with significantly disordered sleep that were more active in task-negative periods than task-oriented periods in participants with FMI, when compared to participants with FM. It was hypothesized that core DMN areas (ie, cingulate cortex, inferior parietal lobule, medial prefrontal cortex, medial temporal cortex, precuneus) would exhibit increased activity during task-negative periods. Analyses revealed that significantly disordered sleep significantly contributed to group differences in the right cingulate gyrus, left lentiform nucleus, left anterior cingulate, left superior gyrus, medial frontal gyrus, right caudate, and the left inferior parietal lobules. Results suggest that FMI may alter some brain areas of the DMN, above and beyond FM. However, future work will need to investigate these results further by controlling for chronic insomnia only before conclusions can be made regarding the effect of FMI comorbidity on the DMN.
\end{abstract}

Keywords: insomnia, fibromyalgia, neuroimaging, task-negative, brain activity, comorbidity

\section{Introduction}

Previous studies provide evidence for the need to examine the relationship between fibromyalgia with comorbid chronic insomnia (FMI) and the default mode network $(\mathrm{DMN})$. The DMN, also known as the task-negative network, is a collection of brain regions that are not active when a task is performed or stimuli is presented. More specifically, these regions are active during rest or when a task is not being performed. ${ }^{1}$ Although some research indicates that these regions are active during autobiographical memory tasks or when noticing one's own internal experience, the functions of this brain network remain mostly unknown., Interestingly, it has been suggested that activating the DMN can alter one's perception of pain by initiating the autonomic and antinociceptive networks. ${ }^{4}$

Previous studies have indicated that the brain's DMN is altered in disease states associated with changes to the central nervous system, including chronic pain and insomnia. $^{5-9}$ Chronic pain conditions, and particularly fibromyalgia (FM), are of increased comorbidity with chronic insomnia (ie, complaint of functional impairment due to sleep disturbance, in addition to difficulty with sleep onset or maintenance, at least three times per week for at least 1 month; $75 \%$ comorbidity of FM). ${ }^{10}$ 
However, the effects of this comorbidity on the DMN are yet unknown. Limited results have indicated that the presence of a comorbid condition is associated with increased brain activation in task-oriented networks, beyond that exhibited by a single disease. ${ }^{11,12}$

One cognitive theory that captures both the neuronal alterations and comorbidity exhibited by FM and chronic insomnia is the Cognitive Activation Theory of Stress (CATS). It posits that sustained psychophysiological stress due to bothersome symptoms (eg, pain, disturbed sleep) sensitizes the central nervous system, causing disorganization, or hyperarousal, of the brain in task-oriented and DMN activity. ${ }^{13,14}$ Although previous studies have provided evidence that increased tasknegative activity is associated with both chronic insomnia and FM, ${ }^{7-9}$ hyperarousal of the DMN has not been examined specifically in those with FMI. Further examination of this comorbidity may provide useful information in the etiology and treatment of these conditions.

The present study was a preliminary investigation of DMN differences in individuals with FM and FMI. This project questioned whether additional disease states, such as sleep disturbance meeting criteria for a diagnosis of chronic insomnia, would be associated with further brain activity alterations. Specifically, novel imaging analyses were utilized to identify regions associated with disordered sleep (ie, significantly decreased total sleep time, significantly increased total wake time) that were more active in task-negative periods than during task-oriented periods in participants with FMI, when compared to participants with FM. It was hypothesized that core DMN areas (ie, cingulate cortex, inferior parietal lobule, medial prefrontal cortex, medial temporal cortex, precuneus $)^{15}$ would exhibit increased activity, associated with significantly disordered sleep, in individuals with FMI, compared to individuals with FM, during task-negative periods in contrast to task-oriented periods.

\section{Methods}

\section{Recruitment}

The present study is a secondary data analysis of a larger study investigating the mechanisms underlying FMI and the potential of cognitive-behavioral treatments for sleep and pain to manipulate those mechanisms. For that study, individuals with FM completed an extensive baseline screening process that included 2 weeks of sleep diaries to determine whether they also met diagnostic criteria for comorbid chronic insomnia. The present study included individuals with FMI (ie, those who qualified for the parent study) as well as those who met criteria for FM, but not comorbid chronic insomnia (ie, those who were disqualified from the parent trial).

Adult participants were recruited from Gainesville and surrounding areas through radio, newspaper, and television advertisements. Criteria for FM were consistent with the recommendations of the American College of Rheumatology. ${ }^{16}$ Criteria for chronic insomnia were consistent with those of the International Classification of Sleep Disorders - Second Edition, ${ }^{17}$ the Diagnostic and Statistical Manual of Mental Disorders - Fourth Edition, Text Revision, ${ }^{18}$ and research diagnostic criteria for chronic insomnia. ${ }^{19,20}$ Inclusion criteria for the study were: a) age 18 or above; b) individual reports currently diagnosed with FM; c) FM confirmed by tender point test: with application of $4 \mathrm{~kg}$ force using a dolorimeter, participant reported pain in at least eleven of 18 points, including points in all four body quadrants (Wagner Force One FDIX Dolorimeter; Wagner Instruments, Greenwich, $\mathrm{CT}$, USA); ${ }^{16} \mathrm{~d}$ ) individual reports of chronic insomnia (ie, sleep onset or awake time during night $>30$ minutes) at least three nights per week for more than 6 months; e) sleep diary confirms insomnia (ie, sleep onset or awake time during night $>30$ minutes) at least six nights during 2-week baseline period; f) daytime dysfunction due to chronic insomnia (ie, mood, cognitive, social or occupational impairment); g) no prescribed or over-the-counter sleep medication for at least 1 month, or stabilized on medication for at least 6 months; h) willing to undergo randomization; and i) able to read and understand English.

Exclusionary criteria for the study were: a) major psychopathology (eg, psychotic disorders, substance abuse); b) a seizure or bipolar disorder; c) other sleep disorders (eg, sleep apnea, periodic limb movements); d) cognitive impairment based on Mini-Mental State Exam ${ }^{21}$ score lower than 23 ( $>9$ th grade education) or 19 (<9th grade education); ${ }^{22}$ e) apneahypopnea index greater than $15 /$ hour or between $10-15 /$ hour with oxygen saturation below $88 \%$; f) periodic limb movement during sleep (PLMS) index greater than 15/hour (AURA Portable Recording System; Grass Technologies [Natus neurology, Middleton, WI, USA]). All records were scored by a registered polysomnographic technologist and reviewed by a physician board certified in sleep medicine. The project coordinator conducted initial telephone screening interviews. Doctoral students in the University of Florida's American Psychological Association accredited clinical psychology program conducted clinical interviews using structured and semi-structured instruments. A licensed clinical psychologist certified in behavioral sleep medicine supervised all screening interviews and confirmed final insomnia diagnoses. 


\section{Sample}

Thirty-nine female participants participated in this study (FMI $n=26, F M n=13$ ). See Table 1 for demographics information by group. Participants were compensated US $\$ 100$ for their participation. The University of Florida's Institutional Review Board approved the study, and prior to participation, all participants signed informed consent forms.

\section{Measures}

\section{Sleep diaries}

Subjective sleep quantity was measured using sleep diaries. The sleep diary ${ }^{23}$ was completed by each participant each morning for 14 days. The sleep diary collected participants' bedtime, wake time (ie, time of last awakening), rise time (ie, time out of bed for the final time), nap time (ie, total time spent napping), sleep onset latency (ie, minutes taken to fall asleep), wake after sleep onset (ie, minutes spent awake after initially falling asleep and before wake time), and snooze time (ie, minutes from wake time to rise time). Two sleep variables were derived from this data: a) total wake time and b) total sleep time. Total wake time was computed by adding sleep onset latency, wake after sleep onset, and snooze time and total sleep time was computed by subtracting total wake time from time in bed (ie, amount of time between bedtime and rise time). Only total wake time and total sleep time were analyzed in the present study. Each participant's 14-day mean was computed for each variable.

\section{Clinical pain}

On the sleep diaries (described above), participants also provided morning and bedtime ratings of current clinical

Table I Group demographic and sleep variables

\begin{tabular}{|c|c|c|}
\hline Variables & $F M(n=13)$ & FMI $(n=26)$ \\
\hline \multicolumn{3}{|l|}{ Demographics } \\
\hline Age (years) & $47.15(13.82)$ & $53.3(14.05)$ \\
\hline \multicolumn{3}{|l|}{ Race } \\
\hline Caucasian (\%) & 76.92 & 80.77 \\
\hline African American (\%) & 15.38 & 11.54 \\
\hline Hispanic (\%) & 7.69 & 7.69 \\
\hline At least some college education (\%) & 76.92 & 65.38 \\
\hline Married (\%) & 53.85 & 42.31 \\
\hline $\mathrm{BDI}$ & $|5.3|(\mid 0.80)$ & $12.88(8.52)$ \\
\hline STAI & $42.31(14.30)$ & $43.19(12.54)$ \\
\hline Clinical pain & $42.78(18.86)$ & $49.47(19.33)$ \\
\hline \multicolumn{3}{|l|}{ Sleep } \\
\hline TST & $468.82(62.37)$ & $403.35(93.75)$ \\
\hline TWT & $69.52(28.78)$ & I $20.83(4 \mid .32)$ \\
\hline
\end{tabular}

Note: Standard deviations are in parentheses.

Abbreviations: FM, fibromyalgia; FMI, fibromyalgia with comorbid chronic insomnia; BDI, Beck Depression Inventory; STAI, State-Trait Anxiety Inventory; TST, total sleep time; TWT, total wake time. pain intensity and pain unpleasantness for 14 days. Ratings were made using visual analog scales with anchors of "no pain sensation" and "most intense pain imaginable" for pain intensity and anchors of "not at all unpleasant" and "most unpleasant imaginable" for pain unpleasantness. The visual analog scales were instantiated on paper as a $10 \mathrm{~cm}$ horizontal line with the two anchors, and participants indicated their pain level by marking a spot on the line. To obtain a numerical score, a ruler was used to measure the distance (in centimeters, to the tenth decimal) between the mark and the left end of the line. The daily values were averaged to get one rating of clinical pain per person. ${ }^{24}$

\section{Mood}

The Beck Depression Inventory Second Edition and StateTrait Anxiety Inventory-Form Y1 were completed prior to neuroimaging. The Beck Depression Inventory Second Edition $^{25}$ is a 21 -item self-report inventory that measures the severity of depressive symptomatology on a three-point scale ranging from 0 (ie, absence of symptoms) to 3 (ie, most severe). Total scores range from 0 to 63 . Ranges for clinical levels of depression are 0 to 13 (ie, minimal), 14 to 19 (ie, mild), 20 to 28 (ie, moderate), and 29 to 63 (ie, severe). The Beck Depression Inventory Second Edition has demonstrated adequate reliability and validity.

The State-Trait Anxiety Inventory-Form $Y 1^{26}$ asks respondents to rate how true 20 self-descriptive statements (eg, "I feel calm") are on a 4-point scale ranging from 1 (ie, not at all) to 4 (ie, very much so). Total scores range from 20 to 80 , with higher scores indicating greater maladjustment. The State-Trait Anxiety Inventory-Form Y1demonstrates test-retest reliability exceeding correlations of 0.7 and reliably distinguishes patient and normal groups. To match the 14-day assessment period for sleep and pain, the typical instructions for both measures were modified to ask participants to respond based on how they generally felt over the preceding 2 weeks.

\section{Experimental paradigm}

Participants underwent a single night of ambulatory polysomnography and were instructed to complete a sleep diary each morning for 14 days prior to completing the neuroimaging protocol. For the neuroimaging protocol, a computer-controlled Medoc Thermal Sensory Analyzer (Ramat Yishai, Israel) delivered trains of eight suprathreshold heat stimuli via a contact thermode $(30 \times 30 \mathrm{~mm})$ placed on the ball of the participant's right foot. Each heat stimulus started at a baseline temperature of $41^{\circ} \mathrm{C}$, peaked at $49.5^{\circ} \mathrm{C}$, then returned to baseline with a rise and decline rate of 
$10^{\circ} \mathrm{C} /$ second. The duration of each stimulus was approximately 1 second with a 3 -second interval separating the peak of each stimulus. ${ }^{27}$

The neuroimaging protocol included four functional runs, lasting approximately 5 minutes and spaced 2 minutes apart. Each included alternating task-oriented periods (ie, eight 1 -second thermal pulses, ranging from warm to painful, received under the ball of the right foot ${ }^{27}$ and task-negative periods (ie, no stimuli provided). Participants were not asked to respond to the thermal stimuli provided during the taskoriented periods or given instructions regarding how to utilize their attention during task-oriented or task-negative periods. A run consisted of an initial 40-second task-negative period, followed by three cycles of a 30 -second task-oriented period and a 60-second task-negative period, and ended with an additional, final 60-second task-negative period.

\section{Functional magnetic resonance imaging (fMRI) acquisition and preprocessing}

fMRI data were acquired using an 8-channel sense head coil. The functional images were obtained from a T2 gradient echo planar imaging sequence which captured 38 contiguous transverse slices of the whole brain parallel to the anterior commissure-posterior commissure plane. Image pre-processing involved: motion correction using trilinear interpolation, slice-scan time correction with sinc interpolation, spatial smoothing with a $4 \mathrm{~mm}$ full-width at half maximum Gaussian kernel, voxel-wise linear detrending, and high-pass temporal filtering to remove non-linear drifts below $3 \mathrm{~Hz}$. The functional images were co-registered to a high-resolution $3 \mathrm{D}$ anatomic volume and transformed into standard Talairach space. ${ }^{28}$ During spatial transformation, functional voxels were interpolated to a resolution of $1 \mathrm{~mm}$.

\section{Functional data analysis}

Data were analyzed with Brain Voyager (BVQX, Version 2.3-Brain Innovation; Maastricht, the Netherlands [http:// brainvoyager.com]) and a MATLAB_r2011b (MathWorks Inc., Natick, MA, USA) toolbox called NeuroElf (created by Jochen Weber; Columbia University, New York, NY, USA [http://www.neuroelf.net]). A random-effects analysis of covariance (ANCOVA) was performed to identify brain regions with: 1) significantly greater task-negative brain activity when compared to brain activity during task-oriented periods, 2) a significant group difference of greater task-negative brain activity in participants with FMI compared to participants with FM, and 3) a significant contribution of disordered sleep (ie, significantly decreased total sleep time, significantly increased total wake time) to predict previous group differences. As a precaution against Type I error, we used two criteria: 1) statistical significance $(P \leq 0.03)$ and 2$)$ minimum cluster size $(135 \mu \mathrm{L})$, the combination of which establishes a probability of a false positive at $0.0007 .{ }^{29}$ The statistical parameter maps were then overlaid on a standardized 3D anatomical volume for localization.

\section{Results}

\section{Independence of sleep disturbance indicators}

Results revealed a significant, negative correlation between total sleep time and total wake time across groups, $r(37)=-0.44$, $P<0.01$, which is within criteria for independence and examination as separate predictors. ${ }^{30}$ Specifically, the results confirmed that total sleep time and total wake time did in fact measure different aspects of sleep disturbance.

\section{Between group heterogeneity}

Independent $t$-tests were run to verify that the sleep variable means of the two groups were significantly different to ensure group task-negative brain activity differences could be attributed to the comorbid insomnia condition. As expected, the groups did significantly differ on sleep as individuals with FMI had significantly less total sleep time (ie, slept less during the night on average) and significantly more total wake time (ie, more fragmentation of sleep on average) than individuals with FM. See Table 2 for mean, standard deviations, and statistical results.

\section{Between group homogeneity}

Between group homogeneity was tested with independent $t$-tests to verify the groups did not differ on clinical pain and affective disturbance (ie, depression, anxiety) to ensure group task-negative brain activity differences could be attributed to sleep variables. The groups did not have significantly different clinical pain ratings, depression, or anxiety. Between group homogeneity was also tested for

Table 2 Between group heterogeneity of sleep variables

\begin{tabular}{llll}
\hline $\begin{array}{l}\text { Sleep } \\
\text { variables }\end{array}$ & $\begin{array}{l}\mathbf{M} \text { and }(\mathbf{S D}) \text { of } \\
\text { FM }(\mathbf{n}=1 \mathbf{1 3})\end{array}$ & $\begin{array}{l}\mathbf{M} \text { and }(\mathbf{S D}) \text { of } \\
\text { FM }(\mathbf{n}=\mathbf{2 6})\end{array}$ & $\boldsymbol{t}(\mathbf{I})$ \\
\hline TST & $468.82(62.37)$ & $403.35(93.75)$ & $-2.27^{*}$ \\
TWT & $69.52(28.78)$ & $120.83(41.32)$ & $4.0 I^{* *}$ \\
\hline
\end{tabular}

Notes: $* P<0.05, * * P=0.001$.

Abbreviations: FM, fibromyalgia only; FMl, fibromyalgia with comorbid chronic insomnia; M, mean; TST, total sleep time; TWT, total wake time. 
Table 3 Between group homogeneity of pain, mood, and demographic variables

\begin{tabular}{|c|c|c|c|c|}
\hline Variables & $F M(n=13)$ & FMI $(n=26)$ & Test result & Significance and effect size \\
\hline Clinical pain & $42.78(18.86)$ & $49.47(19.33)$ & $t(37)=1.03$ & $P=0.3 I, d=0.35$ \\
\hline Depression & $|5.3|(10.80)$ & $12.88(8.52)$ & $t(37)=-0.77$ & $P=0.45, d=0.25$ \\
\hline Anxiety & $42.31(14.30)$ & $43.19(12.54)$ & $t(36)=0.11$ & $P=0.91, d=0.07$ \\
\hline Age & $47.15(13.82)$ & $53.3(14.05)$ & $t(37)=1.30$ & $P=0.20, d=0.44$ \\
\hline $\begin{array}{l}\text { Education (at least some } \\
\text { college education) }\end{array}$ & $76.92 \%$ & $65.38 \%$ & $\mathrm{FET}=3.27$ & $P=0.92, V=0.28$ \\
\hline Race & - & - & $\mathrm{FET}=0.47$ & $P=1.0, V=0.06$ \\
\hline Marital status (married) & $53.85 \%$ & $42.31 \%$ & $\mathrm{FET}=6.42$ & $P=0.23, V=0.43$ \\
\hline
\end{tabular}

Notes: $t$-test used for clinical pain, depression, anxiety, and age; Fisher's exact test was used for all other variables. Means are provided with standard deviations in parentheses.

Abbreviations: FM, fibromyalgia; FMI, fibromyalgia with comorbid chronic insomnia; FET, Fisher's exact test; V, Cramér's V.

age, education, race, and marital status to further verify that group task-negative brain activity could be attributed to sleep variables. Specifically, independent $t$-tests were run on age, while Fisher's exact tests were run on education, race, and marital status to correct for violation of minimum cell number assumptions and small sample size. Results showed that the two groups were not significantly different in age, education, race, or marital status. See Table 3 for mean, standard deviations, percentages, and statistical testing results.

\section{Task-negative brain activity group differences}

Brain regions were first identified where comorbid insomnia status was significantly associated with increased task-negative brain activity, which included the right cingulate gyrus, the left superior gyrus, left medial frontal gyrus, right caudate, and two in the left inferior parietal lobule. Additionally, meaningful trends existed in the left anterior cingulate and the left lentiform nucleus (lateral globus pallidus). See Table 4 for coordinate, voxel number, and statistical testing results of brain regions.

Within these brain regions, decreased total sleep time significantly contributed to the previous group differences in the right cingulate gyrus and the left lentiform nucleus (lateral globus pallidus). In addition, increased total wake time significantly contributed to the previous group differences in the left anterior cingulate, left superior gyrus, left medial frontal gyrus, right caudate, and the left inferior parietal lobules. See Figure 1 for visual diagrams of significant brain region clusters and Table 5 for coordinate, voxel number, and statistical testing results by brain region.

\section{Discussion}

The outcomes of this study are among the first to examine the relationship between comorbid disease states and brain activity alterations of the DMN. These results suggest that comorbid chronic insomnia in fibromyalgia may alter some of the core default mode brain regions, above and beyond FM alone. However, future work will need to investigate these results further by controlling for chronic insomnia only before conclusions can be made regarding the effect of FMI comorbidity on the DMN. As predicted, clinically disordered sleep, due to comorbid insomnia status, was associated with increased task-negative brain activation of well-known DMN brain areas including the right cingulate, medial prefrontal gyrus, left inferior parietal lobule, and the left anterior cingulate. ${ }^{15}$ Significantly disordered sleep was also associated with increased task-negative brain activation of the left lentiform nucleus, left superior gyrus, and right caudate.

Table 4 Brain regions where comorbid insomnia status was significantly associated with increased task-negative brain activity

\begin{tabular}{|c|c|c|c|c|c|c|}
\hline & \multirow[t]{2}{*}{ Number of voxels } & \multicolumn{3}{|c|}{ Coordinates } & \multirow[t]{2}{*}{$F(1.29)$} & \multirow[t]{2}{*}{$P$} \\
\hline & & $\mathbf{x}$ & $\mathbf{Y}$ & $\mathbf{Z}$ & & \\
\hline Right cingulate gyrus BA 24 & 126 & 5 & -2 & 24 & 4.02 & $<0.05$ \\
\hline Left superior frontal gyrus BA 6 & 8 & -9 & 9 & 54 & 6.06 & $<0.05$ \\
\hline Left medial frontal gyrus BA 9 & 5 & -18 & 38 & 19 & 5.67 & $<0.05$ \\
\hline Right caudate BA I 3 & 20 & -23 & 20 & 17 & 7.84 & $<0.01$ \\
\hline Left inferior parietal lobule BA 40 & 6 & -51 & -25 & 34 & 7.58 & $<0.01$ \\
\hline Left inferior parietal lobule BA 40 & 6 & -60 & -25 & 40 & 5.77 & $<0.05$ \\
\hline Left anterior cingulate BA 24 & 5 & -4 & 31 & 10 & 3.30 & 0.08 \\
\hline Left lentiform nucleus & 65 & -22 & -4 & I & 3.53 & 0.07 \\
\hline
\end{tabular}

Note: Coordinates of center voxel for significant clusters. 


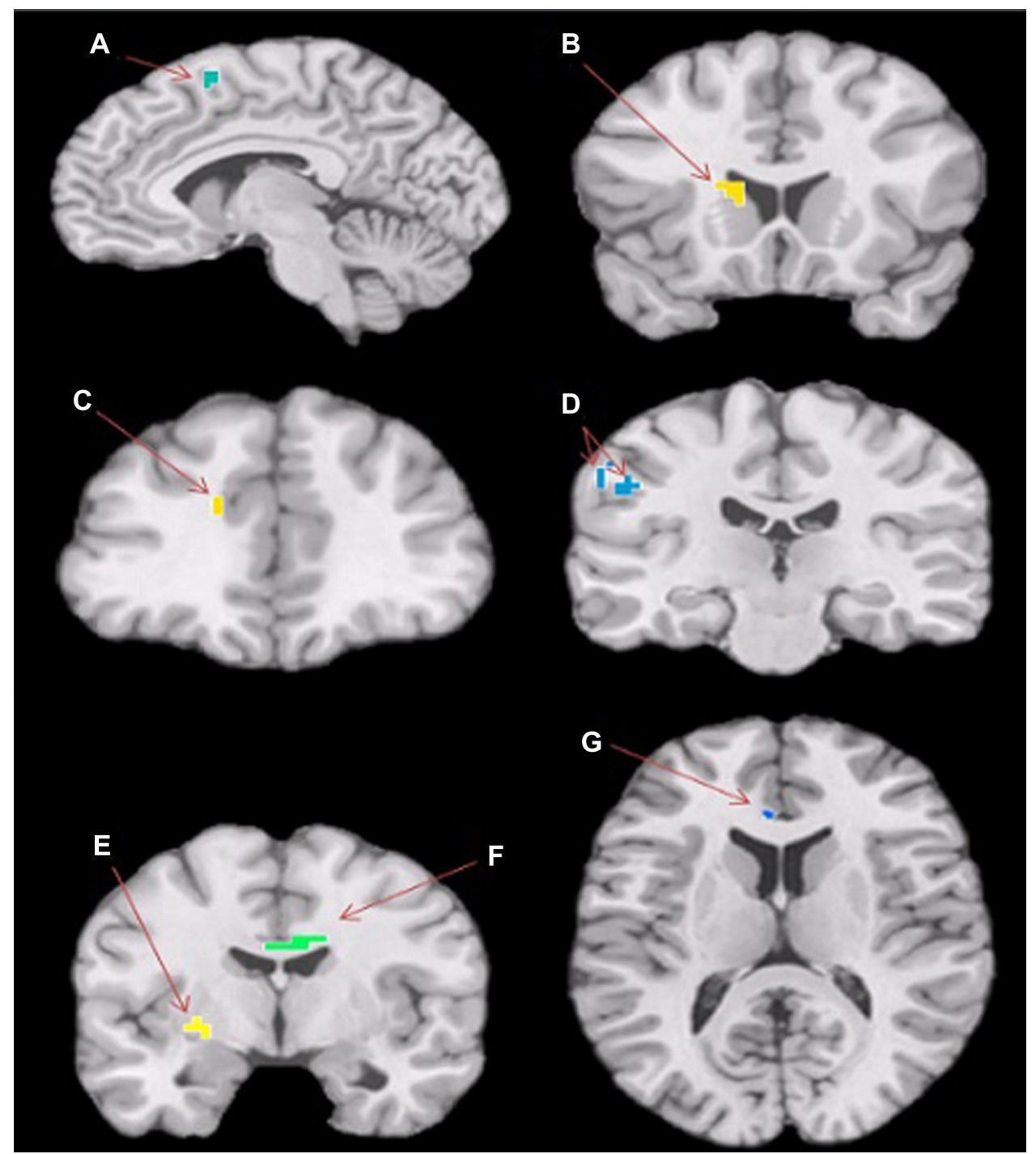

Figure I Brain areas associated with significant group difference (ie, greater task-negative activity in participants with FMI compared to those with FM) and significant contribution of disordered sleep to predict group difference.

Notes: Brain areas are as follows, A) left superior frontal gyrus associated with total wake time B) right caudate associated with total wake time. C) left medical frontal gyrus associated with total wake time. D) left inferior parietal lobules associated with total wake time. E) left lentiform nucleus associated with total sleep time, F) right cingulate gyrus associated with total sleep time, $\mathbf{G}$ ) left anterior cingulate associated with total wake time.

Abbreviations: FM, fibromyalgia; FMI, fibromyalgia with comorbid chronic insomnia.

Within the Cognitive Activation Theory of Stress, chronic pain related alterations to the DMN may place the brain at increased vulnerability to develop chronic insomnia. ${ }^{13}$ Over time, chronic elevated pain response may lead to critical changes in the brain function of the central nervous system that increase the likelihood of further dysregulation of default mode activity. ${ }^{13,31}$ The increased task-negative brain activation noted in the present study within the comorbid condition may suggest an additive effect of alterations to the DMN associated with chronic insomnia status. Specifically, separate amounts of increased activation may be attributed to FM and to chronic insomnia, such that comorbid chronic insomnia is associated with further increases above and beyond
FM-related hyperarousal of these brain regions. As chronic insomnia and FM can be treated using cognitive behavioral therapy methods, ${ }^{32-34}$ future research is needed to test whether the increased brain activation concomitant with FMI can be altered ("corrected/reverted towards normal") by cognitive behavioral therapies.

Contrary to previous studies, ${ }^{35-37}$ pain intensity symptoms were not significantly different across the FM and FMI groups, despite differences in the severity of disordered sleep (ie, sleep disturbance vs clinical diagnosis of chronic insomnia), which allowed pain symptoms to be controlled for when interpreting the present results. Previous studies examined the relationship between pain and sleep quality (self-report) or 
Table 5 Brain regions where total wake time or total sleep time significantly contributed to group differences

\begin{tabular}{|c|c|c|c|c|c|c|}
\hline & \multirow[t]{2}{*}{ Number of voxels } & \multicolumn{3}{|c|}{ Coordinates } & \multirow[t]{2}{*}{$t(1)$} & \multirow[t]{2}{*}{$P$} \\
\hline & & $\mathbf{x}$ & $\mathbf{Y}$ & $\mathbf{Z}$ & & \\
\hline Right cingulate gyrus BA 24 & 126 & 5 & -2 & 24 & 4.94 & $<0.0001$ \\
\hline Left lentiform nucleus & 65 & -22 & -4 & I & 4.30 & $<0.0005$ \\
\hline Left anterior cingulate BA 24 & 5 & -4 & 31 & 10 & 2.41 & $<0.05$ \\
\hline Left superior frontal gyrus BA 6 & 8 & -9 & 9 & 54 & 2.57 & $<0.05$ \\
\hline Left medial frontal gyrus BA 9 & 5 & -18 & 38 & 19 & 2.72 & $<0.01$ \\
\hline Right caudate BA I3 & 20 & -23 & 20 & 17 & 4.25 & $<0.0005$ \\
\hline Left inferior parietal lobule BA 40 & 6 & -51 & -25 & 34 & 2.58 & $<0.05$ \\
\hline Left inferior parietal lobule BA 40 & 6 & -60 & -25 & 40 & 2.94 & $<0.01$ \\
\hline
\end{tabular}

Note: Coordinates of center voxel for significant clusters.

retrospective-questionnaire (ie, Insomnia Severity Index ${ }^{38}$ ). The present investigation differed by separating sleep disturbance from sleep disturbance severe enough to meet criteria for a diagnosis of chronic insomnia based on daily report of minutes needed to fall asleep and time awake after sleep onset over a 2-week period, consistent with the International Classification of Sleep Disorders - Second Edition, ${ }^{17}$ the Diagnostic and Statistical Manual of Mental Disorders Fourth Edition, Text Revision, ${ }^{18}$ and research diagnostic criteria for chronic insomnia. ${ }^{19,20}$ All participants, FM and FMI, complained of a sleep problem based on self-report of sleep quality, but only FMI participants met diagnostic criteria for chronic insomnia.

There are a few limitations that need to be acknowledged. The unequal size of the groups may have reduced the power of the statistical analyses to detect smaller effects. Instead of continuous resting state data where fMRI protocols would not include any task-oriented periods, the fMRI scans in the present study alternated between periods of task-negative and task-oriented stimulation. Although task-oriented periods and dissipation time were controlled for, task-negative periods may be different than traditional resting state as participants were not provided instructions regarding how to focus their attention. A chronic insomnia only group was not included in the study, which is a needed control before conclusions can be made regarding the effects of FMI comorbidity on the DMN. Future research should include both a fibromyalgia only and chronic insomnia only group to further determine comorbid differences.

This preliminary study suggests that future studies should examine these additive alterations to the DMN using a resting brain paradigm in individuals with FMI when compared to individuals with FM only and chronic insomnia only. It is hoped that future studies will continue to build on previous results and reveal increased alterations to the DMN with increased comorbidity when controlling for both FM only and chronic insomnia only. In addition, future research should examine the unique mechanisms behind the increased comorbidity of FMI.

\section{Conclusion}

The outcomes of this study are significant as they are the first to examine the relationship between a comorbid disease, known to alter central nervous system activity, and brain activity alterations of the DMN. The present results of this study suggest that long-term, comorbid pain and sleep disturbance may be associated with increased activation in core default mode brain areas that is above and beyond long-term pain disturbance alone. In addition, comorbid long-term pain and sleep disturbance may be associated with reorganization of the DMN, as additional brain regions appeared to be recruited during task-negative periods. However, future work will need to investigate these results further by controlling for chronic insomnia only before conclusions can be made regarding the effect of FMI comorbidity on the DMN. Within the Cognitive Activation Theory of Stress, chronic pain related alterations to the DMN may place the brain at increased vulnerability to develop chronic insomnia. ${ }^{13}$ Future work should examine neural changes in patients with FMI following cognitive behavioral therapies for pain and insomnia to examine whether the effectiveness of cognitive behavioral therapies is related to "normalization" of the DMN.

\section{Acknowledgments}

Funding: study support was provided by Award Number R01AR055160 and R01AR055160-S1 ARRA supplement from the National Institute of Arthritis And Musculoskeletal And Skin Diseases (Christina S McCrae, principal investigator; Michael E Robinson, co-principal investigator). Thanks are given to Christine Towler (lab coordinator); Brittany Telford, Zac Zedar (polysomnography assistants); Susan Purdy 
(registered polysomnographic technologist); and Janelle Letzen, (neuroimaging support) for their contributions to this research project.

\section{Disclosure}

The authors have no conflicts of interest to report.

\section{References}

1. Raichle ME, MacLeod AM, Snyder AZ, Powers WJ, Gusnard DA, Shulman GL. A default mode of brain function. Proc Natl Acad Sci US A. 2001;98(2):676-682.

2. Zysset S, Huber O, Ferstl E, von Cramon DY. The anterior frontomedian cortex and evaluative judgment: an fMRI study. Neuroimage. 2002; 15(4):983-991.

3. Buckner RL, Snyder AZ, Shannon BJ, et al. Molecular, structural, and functional characterization of Alzheimer's disease: evidence for a relationship between default activity, amyloid, and memory. J Neurosci. 2005;25(34):7709-7717.

4. Kucyi A, Salomons TV, Davis KD. Mind wandering away from pain dynamically engages antinociceptive and default mode brain networks. Proc Natl Acad Sci U S A. 2013;110(46):18692-18697.

5. Baliki MN, Geha PY, Apkarian AV, Chialvo DR. Beyond feeling: chronic pain hurts the brain, disrupting the default-mode network dynamics. J Neurosci. 2008;28(6):1398-1403.

6. LiY, Dou S, Wang E, et al. Evaluation of brain default network fMRI of insomnia with depression patients at resting state. Life Science Journal. 2014;11(8):794-801.

7. Huang Z, Liang P, Jia X, et al. Abnormal amygdala connectivity in patients with primary insomnia: evidence from resting state fMRI. Eur J Radiol. 2012;81(6):1288-1295.

8. Harris RE. Elevated excitatory neurotransmitter levels in the fibromyalgia brain. Arthritis Res Ther. 2010;12(5):141.

9. Napadow V, LaCount L, Park K, As-Sanie S, Clauw DJ, Harris RE. Intrinsic brain connectivity in fibromyalgia is associated with chronic pain intensity. Arthritis Rheum. 2010;62(8):2545-2555.

10. Peres MF, Young WB, Kaup AO, Zukerman E, Silberstein SD. Fibromyalgia is common in patients with transformed migraine. Neurology. 2001;57(7):1326-1328.

11. Hassel S, Almeida JR, Frank E, et al. Prefrontal cortical and striatal activity to happy and fear faces in bipolar disorder is associated with comorbid substance abuse and eating disorder. $J$ Affect Disord. 2009; $118(1-3): 19-27$.

12. Waugh CE, Hamilton JP, Chen MC, Joormann J, Gotlib IH. Neural temporal dynamics of stress in comorbid major depressive disorder and social anxiety disorder. Biol Mood Anxiety Disord. 2012;2(1):11.

13. Eriksen HR, Ursin H. Sensitization and subjective health complaints. Scand J Psychol. 2002;43(2):189-196.

14. Eriksen HR, Ursin H. Subjective health complaints, sensitization, and sustained cognitive activation (stress). J Psychosom Res. 2004;56(4): 445-448.

15. Buckner RL, Andrews-Hanna JR, Schacter DL. The brain's default network: anatomy, function, and relevance to disease. Ann N Y Acad Sci. 2008;1124:1-38.

16. Wolfe F, Smythe HA, Yunus MB, et al. The American College of Rheumatology 1990 Criteria for the Classification of Fibromyalgia. Report of the Multicenter Criteria Committee. Arthritis Rheum. 1990;33(2):160-172.

17. American Sleep Disorders Association. The International Classification of Sleep Disorders: Diagnostic and Coding Manual. Chicago, IL: American Sleep Disorders Association; 1997.

18. American Psychiatric Association. Diagnostic and statistical manual of mental disorders fourth edition text revision. Washington, DC: American Psychiatric Association; 2000.
19. Edinger JD, Bonnet MH, Bootzin RR, et al. Derivation of research diagnostic criteria for insomnia: report of an American Academy of Sleep Medicine Work Group. Sleep. 2004;27(8):1567-1596.

20. Lichstein KL, Durrence HH, Taylor DJ, Bush AJ, Riedel BW. Quantitative criteria for insomnia. Behav Res Ther. 2003;41(4):427-445.

21. Folstein MF, Folstein SE, McHugh PR. "Mini-mental state". A practical method for grading the cognitive state of patients for the clinician. J Psychiatr Res. 1975;12(3):189-198.

22. Almeida OP. Mini exame dos estado mental e o diagnóstico de demência no Brasil [Mini mental state examination and the diagnosis of dementia in Brazil]. Arq Neuropsiquiatr. 1998;56(3B):605-612. Portuguese.

23. Lichstein KL, Riedel BW, Means MK. Psychological treatment of latelife insomnia. In: Schulz R, Maddox G, Lawton MP, editors. Annual review of gerontology and geriatrics. Vol 18. New York: Springer; 1999:74-110.

24. Price DD, McGrath PA, Rafii A, Buckingham B. The validation of visual analogue scales as ratio scale measures for chronic and experimental pain. Pain. 1983;17(1):45-56.

25. Beck AT, Steer RA, Ball R, Ranieri W. Comparison of Beck Depression Inventories-IA and -II in psychiatric outpatients. J Pers Assess. 1996; 67(3):588-597.

26. Spielberger CD, Gorsuch RL, Lushene R, Vagg PR, Jacobs GA. StateTrait Anxiety Inventory, Form Y. Palo Alto, CA: Consulting Psychologists Press; 1983.

27. Staud R, Craggs JG, Robinson ME, Perlstein WM, Price DD. Brain activity related to temporal summation of C-fiber evoked pain. Pain. 2007;129(1-2):130-142.

28. Talairach J, Tournoux P. Co-Planar Stereotaxic Atlas of the Human Brain. Three-Dimensional Proportional System: An Approach to Cerebral Imaging. Stuttgart: Thieme; 1988.

29. Forman SD, Cohen JD, Fitzgerald M, Eddy WF, Mintun MA, Noll DC. Improved assessment of significant activation in functional magnetic resonance imaging (fMRI): use of a cluster-size threshold. Magn Reson Med. 1995;33(5):636-647.

30. Rubin A. Statistics for Evidence-Based Practice and Evaluation. 3rd ed. Stamford, CT: Brooks Cole; 2012.

31. Wilhelmsen I. Brain-gut axis as an example of the bio-psycho-social model. Gut. 2000;47 Suppl 4:iv5-iv7.

32. Bernardy K, Fuber N, Kollner V, Hauser W. Efficacy of cognitivebehavioral therapies in fibromyalgia syndrome - a systematic review and metaanalysis of randomized controlled trials. J Rheumatol. 2010;37(10):1991-2005.

33. Tang NK, Goodchild CE, Salkovskis PM. Hybrid cognitive-behaviour therapy for individuals with insomnia and chronic pain: a pilot randomised controlled trial. Behav Res Ther. 2012;50(12):814-821.

34. Espie CA, Inglis SJ, Tessier S, Harvey L. The clinical effectiveness of cognitive behaviour therapy for chronic insomnia: implementation and evaluation of a sleep clinic in general medical practice. Behav Res Ther. 2001;39(1):45-60.

35. Nicassio PM, Moxham EG, Schuman CE, Gevirtz RN. The contribution of pain, reported sleep quality, and depressive symptoms to fatigue in fibromyalgia. Pain. 2002;100(3):271-279.

36. Affleck G, Urrows S, Tennen H, Higgins P, Abeles M. Sequential daily relations of sleep, pain intensity, and attention to pain among women with fibromyalgia. Pain. 1996;68(2-3):363-368.

37. Tang NK, Wright KJ, Salkovskis PM. Prevalence and correlates of clinical insomnia co-occurring with chronic back pain. J Sleep Res. 2007;16(1):85-95.

38. Bastien CH, Vallières A, Morin CM. Validation of the Insomnia Severity Index as an outcome measure for insomnia research. Sleep Med. 2001;2(4):297-307. 
Journal of Pain Research

\section{Publish your work in this journal}

The Journal of Pain Research is an international, peer-reviewed, open access, online journal that welcomes laboratory and clinical findings in the fields of pain research and the prevention and management of pain. Original research, reviews, symposium reports, hypothesis formation and commentaries are all considered for publication.

The manuscript management system is completely online and includes a very quick and fair peer-review system, which is all easy to use. Visit http://www.dovepress.com/testimonials.php to read real quotes from published authors.

\footnotetext{
Submit your manuscript here: http://www.dovepress.com/journal-of-pain-research-journal
} 